VOL. 17 (1977), 298.

\title{
Sums of independent random
}

\section{variables and regular variation}

\section{Ross Arthur Maller}

This thesis is concerned with further investigating, following the work of $W$. Feller, the application of the theory of regular variation, or generalisations of it, to various aspects of the classical theory of the convergence of normed sums of independent and identically distributed random variables. We are concerned, not only with the convergence of (or the convergence of subsequences of) the normed sums in distribution to nondegenerate random variables (this being the situation in the theory of domains of attraction, stochastic compactness and partial attraction treated in Chapters 2 and 3 of this thesis), but we hope to show also (in Chapters 4 and 5) that the theory of regular variation, and some generalisations of it, can prove useful in describing particular kinds of degenerate convergence and almost sure behaviour of such sums. Thus, we look at problems related to relative stability, the strong law of large numbers, and the law of the iterated logarithm.

Chapters 2, 3, 4, and 5 contain our main results, but as well as these we give some related investigations: we consider compactness criteria for triangular arrays, and some local limit, large deviations and rate of convergence problems for sums of independent random variables, these last three applications being closely connected with properties related to the concept of regular variation. In an appendix to Chapter 5 we give a strong law for stationary markovian processes satisfying a uniform mixing condition; the proof of this follows easily from the methods we develop for independent random variables.

Received 26 August 1977. Thesis submitted to the Australian National University, January 1977. Degree approved, August 1977. Supervisors: Dr C.C. Heyde (CSIRO), Dr Eugene Seneta. 